\title{
ISHNE/EHRA Expert Consensus on Remote Monitoring of Cardiovascular Implantable Electronic Devices (CIEDs)
}

\author{
Sergio Dubner,* Angelo Auricchio, $†$ Jonathan S. Steinberg, $\ddagger$ Panos Vardas, $\S$ \\ Peter Stone, ${ }^{* *}$ Josep Brugada, $\dagger \dagger$ Ryszard Piotrowicz, $\nmid \dagger$ David L. Hayes, $\S \S$ \\ Paulus Kirchhof, $* * *, \dagger \dagger \dagger$ Günter Breithardt, $\dagger \dagger \dagger$ Wojciech Zareba, $+\dagger \dagger$ \\ Claudio Schuger,§§§ Mehmet K. Aktas, $\ddagger \ddagger$ Michal Chudzik,**** Suneet Mittal, \\ and Niraj Varmat†††
}

Document reviewers: Carsten Israel (Germany), Luigi Padeletti (Italy), and Michele Brignole (Italy).

From the *Clinica y Maternidad Suizo Argentina, Buenos Aires, Argentina; †Fondazione Cardiocentro Ticino, Lugano, Switzerland; $\ddagger$ Valley Heart and Vascular Institute and Columbia University College of Physicians $\mathcal{E}^{2}$ Surgeons, New York; \$Heraklion University Hospital, Crete, Greece; ** Brigham E̊ Women's Hospital, Boston, Massachusetts; ††Thorax Institute-Hospital Clinic, University of Barcelona, Barcelona, Spain; † National Institute of Cardiology, Warsaw, Poland; $\$$ Mayo Clinic, Rochester, Minnesota; ***University of Birmingham Centre for Cardiovascular Sciences, Birmingham, UK; †††Department of Cardiology and Angiology, University Hospital Münster, Münster, Germany; $甘+$ University of Rochester, Rochester, New York; $\$ \S \S$ Henry Ford Hospital, Detroit, Michigan; ****Department of Electrocardiology, Medical University of Lodz, Poland; and $\dagger \dagger \dagger \dagger$ Cleveland Clinic, Cleveland, Ohio

\begin{abstract}
We are in the midst of a rapidly evolving era of technology-assisted medicine. The field of telemedicine provides the opportunity for highly individualized medical management in a way that has never been possible before. Evolving medical technologies using cardiac implantable devices with capabilities for remote monitoring permit evaluation of multiple parameters of cardiovascular physiology and risk, including cardiac rhythm, device function, blood pressure values, the presence of myocardial ischaemia, and the degree of compensation of congestive heart failure. Cardiac risk, device status, and response to therapies can now be assessed with these electronic systems of detection and reporting. This document reflects the extensive experience from investigators and innovators around the world who are shaping the evolution of this rapidly expanding field, focusing in particular on implantable pacemakers, implantable cardioverter defibrillators, devices for
\end{abstract}

\footnotetext{
Address for correspondence: Sergio Dubner, Clinica y Maternidad Suizo Argentina, Buenos Aires, Argentina. Tel: +541148273654; Fax: +541148274895; E-mail:dubner@fibertel.com.ar

Conflict of interest: S.D. received consulting fees from Boehringer Ingelheim and research grants from Biotronik, St Jude Medical. A.A. received consulting fees from Sorin Group, Medtronic, Biotronik, EBR System, Merk, Biosense, Webster, BDS Cordis, Philips, Impulse Dynamics and speaker fee from Sorin Group, Medtronic, Biotronik, St Jude, Abbott. J.S. received consulting fees from BiosenseWebster, Siemens, St Jude Medical, Sanofi-Aventis, Ortho-McNeil, Medtronic, Ambucor, and research support from Biosense-Webster, Medtronic, Lifewatch. P.V. received consulting fees from Astra Zeneca, Bayer Healthcare, Boehringer Ingelheim, Medtronic, Inc., Sanofi Aventis, Servier, Menarini, and Pfizer, and research grants from Servier, Astra Zeneca, Sanofi Aventis, Medtronic Inc., St Jude Medical. D.L.H. served as a advisory capacity of St Jude Medical, Boston Scientific, Medtronic; speak at Educational venues of St Jude Medical, Boston Scientific, Medtronic, Biotronik and Sorin Medical and also as a Steering committee of St Jude Medical, Medtronic. P.K. received consulting fees from $3 M$ Medica, Astra-Zeneca, Bayer, Boehringer, MEDA Pharma, Medtronic, Merck, MSD, Pfizer/BMS, Sanofi, Servier, Siemmes, Takeda, and research grants from $3 M$ Medica, Cardiovascular Therapeutics, MEDA Pharma, Medtronic, OMRON, Sanofi, St Jude Medical. M.C. received consulting fees from Biotronik and St Jude Medical. S.M. is a consultant to Biotronik, Boston Scientific, Medtronic and St Jude Medical. W.Z. received a significant research grant from Boston Scientific. C.S. received research grants from Medtronic, St Jude, Boston Scientific. N.V. received research grants and consultancies from Biotronik, Boston Scientific, Medtronic, St Jude Medical.
} 
cardiac resynchronization therapy (both with and without defibrillation properties), loop recorders, and hemodynamic monitoring devices. This document covers the basic methodologies, guidelines for their use, experience with existing applications, and the legal and reimbursement aspects associated with their use. To adequately cover this important emerging topic, the International Society for Holter and Noninvasive Electrocardiology and the European Heart Rhythm Association combined their expertise in this field. We hope that the development of this field can contribute to improve care of our cardiovascular patients.

Ann Noninvasive Electrocardiol 2012;17(1):36-56

remote monitoring; cardiovascular implantable electronic devices; ventricular tachycardia/ ventricular fibrillation

\section{INTRODUCTION}

Cardiovascular implantable electronic devices (CIEDs) $)^{1,2}$ have expanded in number and complexity since their introduction in 1958. On the basis of Eucomed data, 395,000 implantable pacemakers (IPGs) and 62,000 implantable cardioverter defibrillators (ICDs) were implanted in European countries included in the Eucomed survey during 2009. ${ }^{3}$ Since some countries are missing in the survey, average overall implantation rates of $947 /$ million for IPGs, 149/million for ICDs, 26/million for cardiac resynchronization therapy (CRT)-Ps, and 85/million CRT-Ds are estimated, which was applied to the total population of "Europe 27," i.e., $497,659.81$ million. This would lead to total implants in 2009 in "Europe 27" of 471,284 IPGs and 74,151 ICDs $^{3}$ (Figs. 1A-C).

This expanding population of patients with implantable cardiac devices requires special care. The devices require regular technical checks and adaptation of their function to the needs of individual patients. Furthermore, implanted devices provide a new source for continuous monitoring of biosignals that may contain relevant medical information.

The logistics of monitoring these devices have already placed a substantial and increasing burden on the cardiovascular community. ${ }^{4}$ In addition, since these are implantable devices, there is an ongoing opportunity and responsibility to manage both the patient and the device.

On the other hand, telecardiology is a growing entity with more and more applications in arrhythmias and device evaluation. During the last decade, it has evolved rapidly from an experimental diagnostic method to its current status; however, there is a lack of information and agreement regarding the application, utility, and reimbursement of telemonitoring through devices.

Cardiac patients represent the largest segment of patients being monitored by wireless telemetry.
Wireless medical telemetry provides access to measurement and recording of physiological parameters and other patient-related information via transmitted electromagnetic signals.

The data downloaded from the device by the transmitter are then sent to the hospital or manufacturer center, either manually or automatically, using either the landline phone or a mobile telecommunications (usually global system for mobile communications [GSM]) network. The information is received, analyzed, and made available to the treating physician. Although it is not an emergency system, it helps to generate a fast response. ${ }^{5}$

Many current CIEDs are able to automatically execute the tests that are performed manually at the outpatient clinic, such as battery status, lead impedances, or sensing and capture thresholds. Data acquired automatically on a predefined periodic basis by the device can then be sent from the patient's home to the physician using the transmitter, thus avoiding an unnecessary in-clinic visit.

Rapid advances in technology and wider availability of patient-friendly equipment give the patient the opportunity to get involved in his own care with unscheduled transmission of any predefined alerts to the physician, and, on the other hand, help the providers of care to identify early signs of worsening heart failure and its precipitating factors or of arrhythmias such as atrial fibrillation (AF). Therefore, remote monitoring (RM) has the potential to offer improved patient safety and quality of care. $^{6}$

The purpose of this position article is to delineate the current status and potential future direction to make effective and efficient use of telemonitoring in implanted devices and to focus on the followup of patients with CIEDs by providing daily information on the device and the performance of the patient to their physicians using wireless communication. This document is intended to describe the medical aspects of these activities, including 


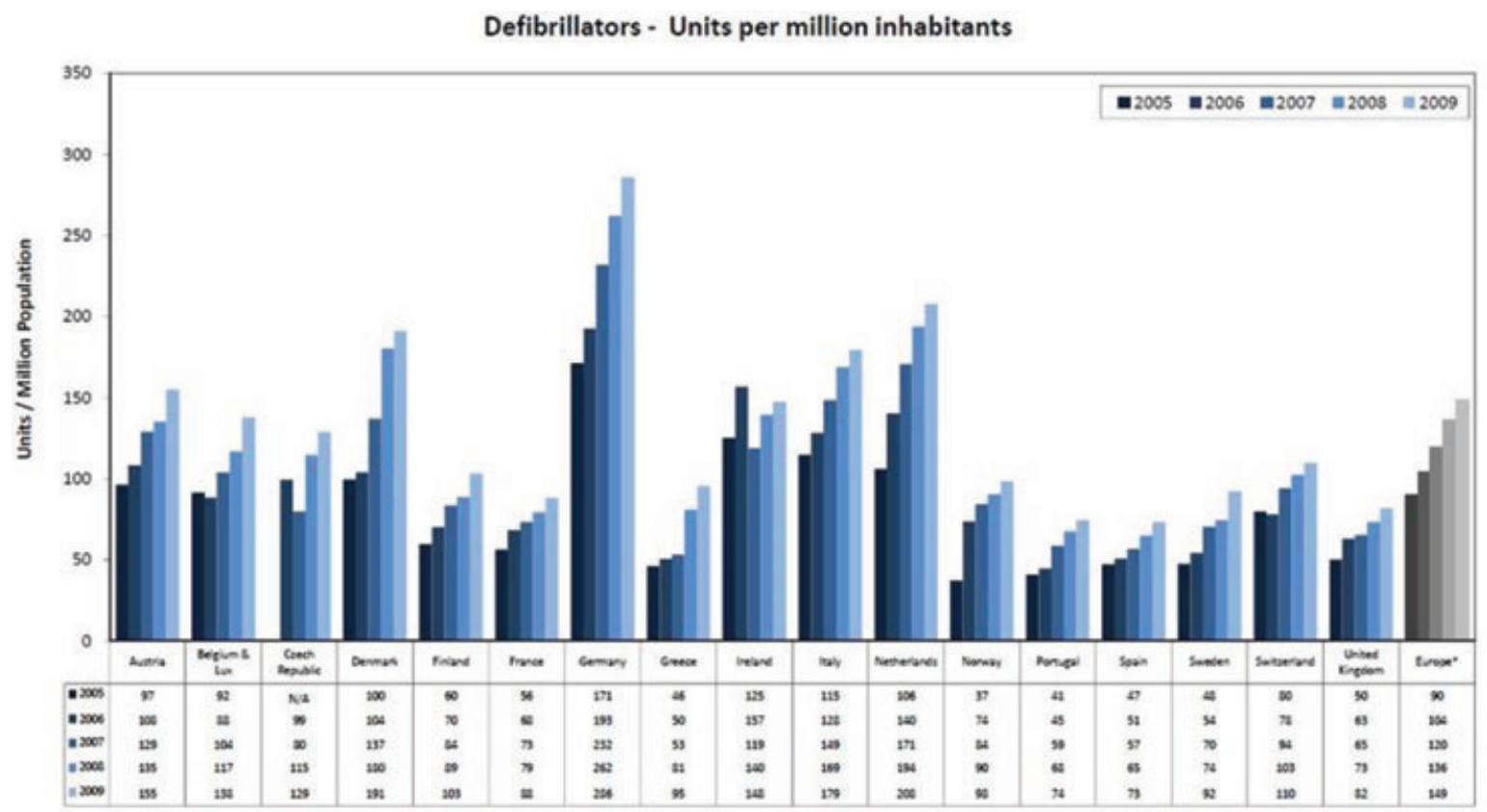

a

Pacemakers - Units per million inhabitants

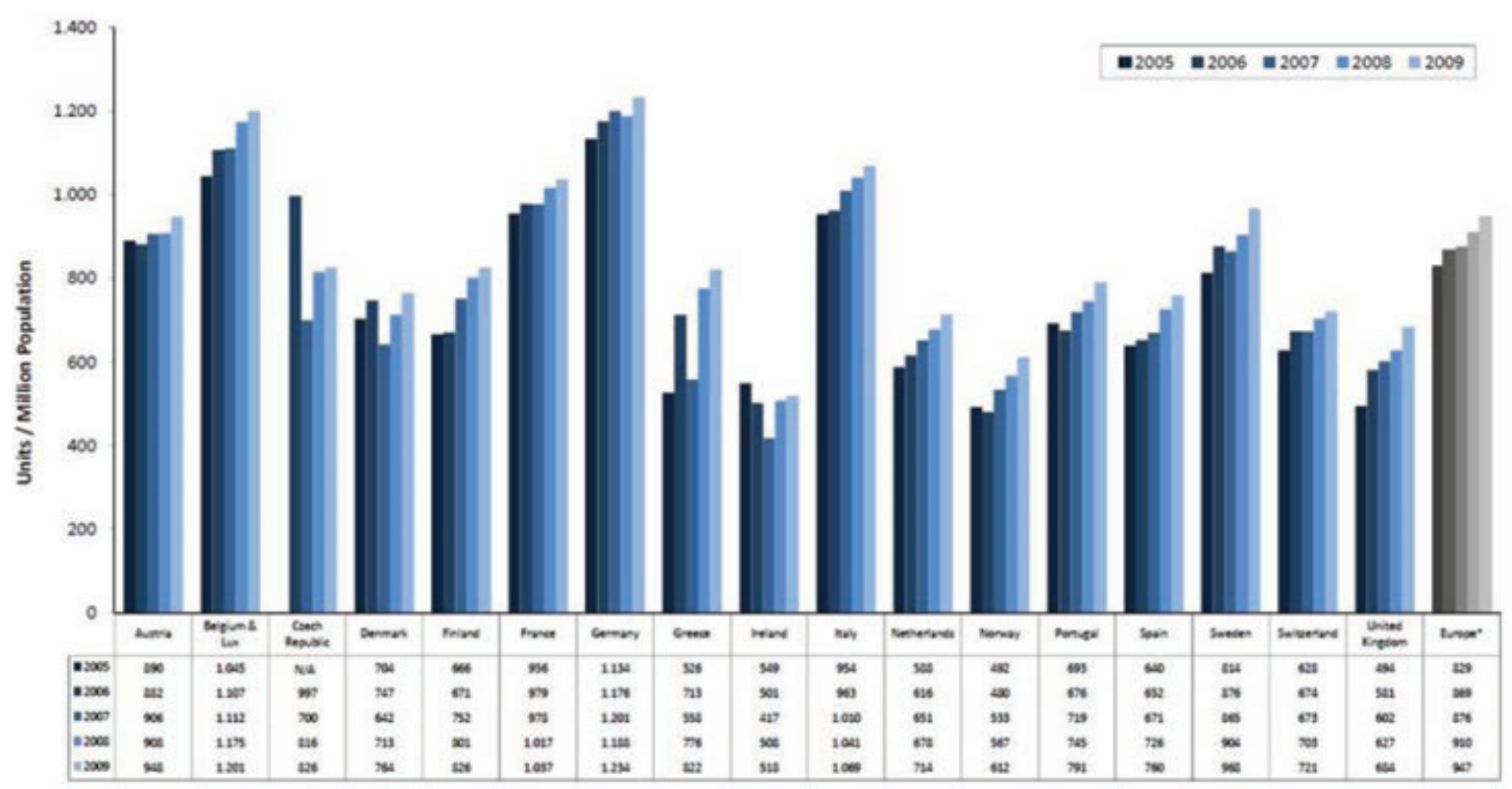

b

Figure 1. (a), (b), and (c) Implanted cardiac devices per million inhabitants in Europe. Reproduced with permission from Eucomed. ${ }^{3}$

definitions, utility, benefits, legal aspects, and reimbursement for telemedicine in CIEDs. The implementation details in telemonitoring and followup will vary in differing geographic locations with diverse medical and governmental structures, but it is the intent to provide suggestions for universally applicable and clinically appropriate monitoring of CIEDs. 

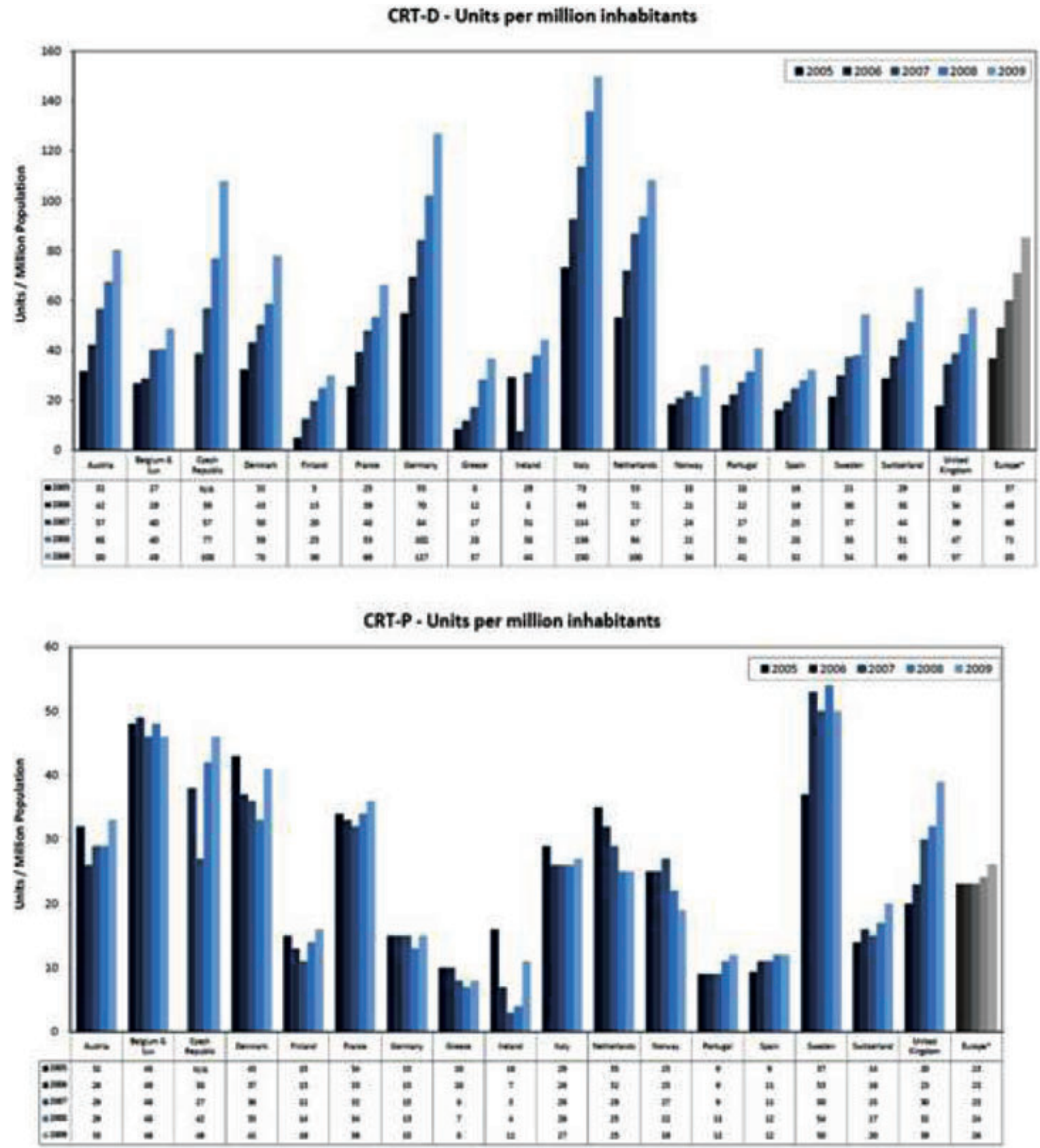

C

Figure 1. (Continued.)

\section{DEFINITIONS}

According to the expert consensus document of Heart Rhythm Society (HRS)/European Heart Rhythm Association (EHRA) on CIEDs monitoring, ${ }^{4}$ the availability of RM and, in the future, remote programming of CIEDs will require a change in CIEDs' follow-up paradigms and protocols.
Remote monitoring technology may reduce faceto-face clinic visits and will provide essential and timely information, when visits are triggered by a clinical event. In addition, RM and the resulting collection of device component long-term reliability data may aid in the assessment of CIEDs' system performance. The system may then act as a warning mechanism for the early discovery of 
Table 1. Definitions

\begin{tabular}{lll}
\hline Equipment & \multicolumn{1}{c}{ Devices } & \multicolumn{1}{c}{ PM-ICD-CRT-ILR } \\
\hline & Devices interrogation & Telemetry to retrieve information \\
& Device programming & Bidirectional telemetry \\
& Programmer & Receive and transmit info via telemetry \\
& Home monitoring & Remote device able to communicate with CIED \\
Remote FU & Remote monitoring & Programmable scheduled transmissions \\
& Remote FU & Automatically unscheduled transmissions \\
& Remote monitoring & Nonscheduled FU as a result of a patient experiencing a real \\
& Pt initiated interrogations & or perceived clinical event \\
\hline
\end{tabular}

potential issues and clinical conditions that may lead to an increased frequency of in-person or remote surveillance.

Our intention is to define the different nomenclature that has been used so far in traditional device follow-up and in the newly developed RM and follow-up, in an effort to standardize the terms normally utilized in the description of the functionality of these devices and their monitoring ability (Table 1).

\section{Equipment}

Devices Currently Amenable of Remote Monitoring

The current CIEDs being interrogated remotely include: $^{7}$ pacemakers (PMs), ICDs, CRTs, implantable loop recorders (ILRs), and implantable hemodynamic monitors.

\section{Device Interrogation}

It uses telemetry to retrieve information on the CIED-programmed parameters and data stored in the CIED memory. These data may be retrieved and stored directly in a CIED programmer, on a dedicated personal computer or retrieved, and then stored remotely on a server to be viewed on an internet website.

\section{Device Programming}

Bidirectional telemetry using encoded and encrypted radiofrequency signals from a programmer allows noninvasive, reversible changes in some of the operating parameters of the CIED that enables the operator to select CIED settings to assess and optimize the system performance and longevity and to tailor these parameters to the individual patient's condition. Because of safety considerations, device programming can only be done by the use of a specific programmer in the setting of a face-toface evaluation.

\section{Programmer}

It is a device designed to receive and transmit information via telemetry from a family of CIEDs. Programmers are manufacturer-specific and will display and print the information to the operator and temporarily or permanently program the behavior of the CIED. Traditionally, programmers communicated with CIEDs via a "wand," attached by a wire to the programmer and positioned on the body's surface over the CIED implantation site to receive the telemetry signal. However, modern devices can be interrogated and programmed "remotely," i.e., without the use of a wand. The distance for radiofrequency communication has increased from several centimeters (2-5 inches) to several meters (10-20 feet). ${ }^{8}$ The ability to remotely communicate with a station has opened the possibility of "in-home monitoring" as it will be defined later. This remote telemetry is device-specific but uses either the Industrial, Scientific, and Medical band from 902 to $928 \mathrm{MHz}$ or a subsection of the Medical Implant and Communications band from 402 to $405 \mathrm{MHz}$.

\section{Home Monitor/Communicator}

This is a remote telemetry device able to communicate with the CIED automatically in real time or at scheduled intervals, and that transmits the encrypted data over long distances utilizing telephone lines or cellular phone technology. The data are then entered and stored in dedicated servers that act as data repositories that communicate actively or passively with the caregivers of the patient. 
Remote Monitoring Systems

Remote monitoring of PMs trans-telephonically (TTM) was introduced in 1971 and remained until recently the main technology to remotely follow the performance of PMs. It is mostly aimed at ascertaining the integrity of the system especially with regard to battery performance and longevity, appropriate capture, and sensing.

With the advent of remote wireless communication from the CIED to a home monitor/ communicator and, in turn, the ability to transmit and store all data that the CIED is capable of collecting, manufacture-specific remote monitor systems proliferated and became the new standard for remote follow-up (Medtronic CareLink, Boston Scientific Latitude, Biotronik Home Monitoring, Sorin Smart View and St Jude Merlin.net). ${ }^{9-13}$ A brief display of their properties is included in Table $2 .{ }^{6}$

\section{Remote Follow-Up and Monitoring Clinical Scenarios}

The circumstances in which this occurs and the protocols to obtain the information and disseminate it to the caregivers vary by manufacturer and the capabilities of each RM system (refer to Table 1). The most common clinical scenarios are defined below.

\section{Remote Follow-Up}

Refers to programmable scheduled transmissions in which routine CIED parameters are collected remotely in a format similar to that obtained during a routine clinic visit. This information obtained by the caregiver from the data repository (usually via the Internet) is encoded in such a way that could be interfaced with commercially available PMs and ICD follow-up software (i.e., Paceart). As opposed to TTM, practically all information available through the face-to-face interrogation with a programmer can be obtained.

\section{Remote Monitoring}

Refers to data acquired automatically with unscheduled transmissions of any prespecified alerts related to device functioning or to clinical events. The latter adds a new functionality to implanted devices, opening a new era of potentially beneficial preemptive interventions that may alter the natural history of a particular disease or condition.

\section{Patient-Initiated Interrogations}

Refers to non-scheduled follow-up interrogations as a result of a patient experiencing a real or perceived clinical event, for which the patient is seeking expert evaluation.

\section{DESCRIPTION OF THE TECHNOLOGY}

Remote telemetry data are transmitted from the device to the home monitoring (HM) station either by a "wand" (remote transmitter monitor $[\mathrm{RTM}]),{ }^{14-16}$ or by wireless communication between device and HM station. In addition to scheduled, planned interrogation and data transmission sessions, automatic, or alert-triggered data (e.g., significant change in lead impedance, development of persistent $\mathrm{AF}$ with fast ventricular rate close to ventricular fibrillation (VF) zone, frequent episodes of ventricular tachycardia (VT), delivery of (the device does not know that shocks were inappropriate) frequent shocks, or changes in hemodynamic status) can be transmitted depending on the device. ${ }^{17,18}$

This home monitor is linked by telephone to a central (internet-based) secure server/secure website automatically to deposit the data for further analysis. Transmission can also occur via an analog phone landline, a digital subscriber line, or a device accessory phone line.

The physician can receive a company alert notification via pager, fax, SMS, voice message, or e-mail. Many systems require access to a dedicated (device-or company-specific) website to obtain detailed information on the interrogation. The physician can activate either manually or automatically message calls to patients to remind them of upcoming remote follow-up appointments, to notify them if they miss a remote follow-up appointment, to ask to call the clinic, to inform that their remote transmissions have been reviewed by the clinic, etc. ${ }^{19,20}$ Reprogramming of CIED by RTM is not yet implemented in clinical practice, mainly due to safety considerations regarding data protection and unauthorized control of device function (Fig. 2).

\section{REVIEW OF EXISTING EVIDENCE FOR DEVICE-BASED MONITORING FOCUSED ON INSERTABLE CARDIAC MONITORS}

Arrhythmia (especially AF) and syncope are two important conditions that should be optimally 


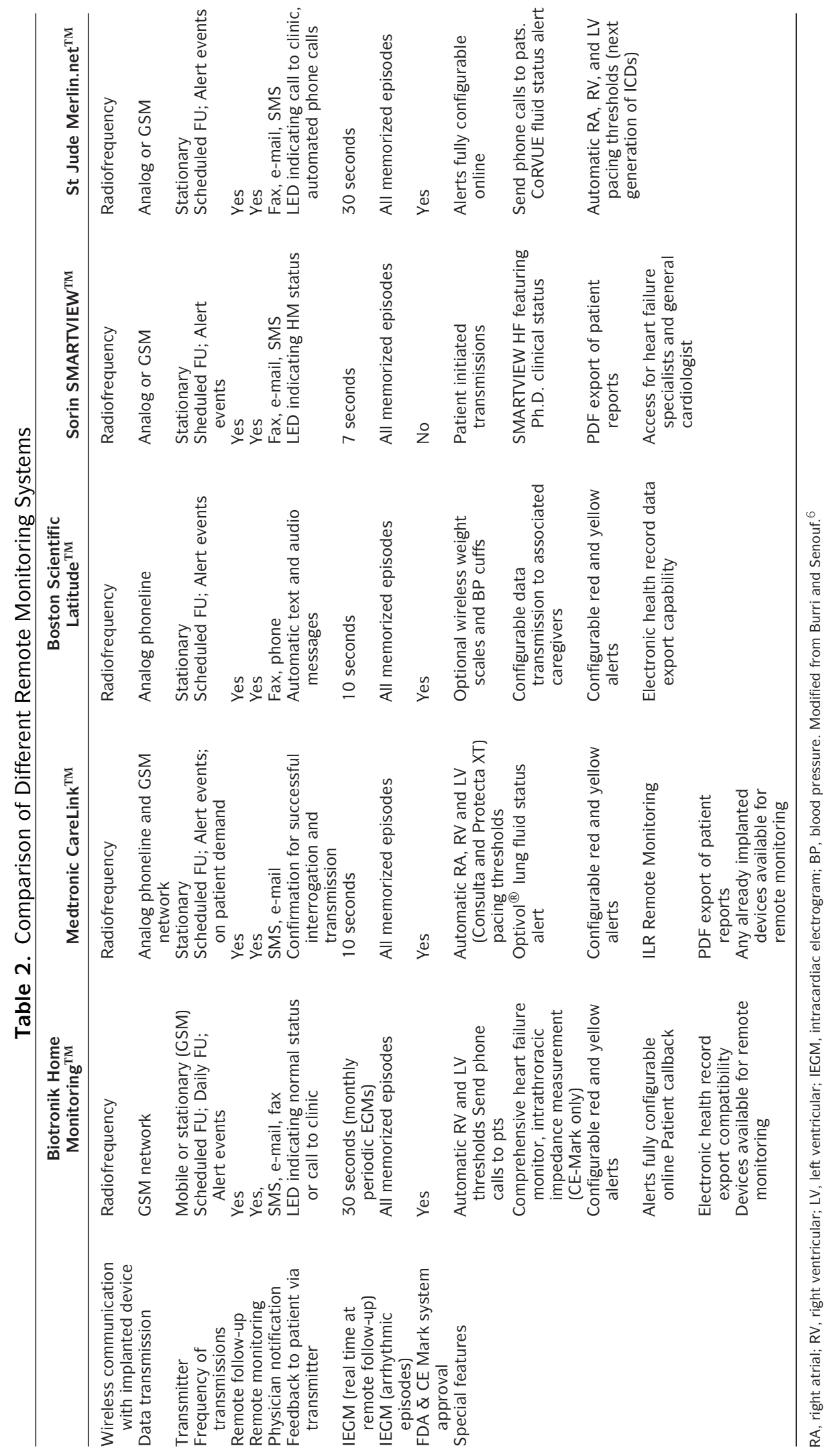




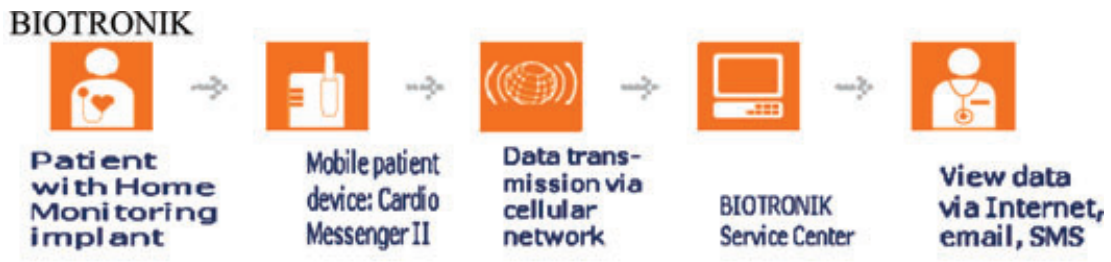

MEDTRONIC

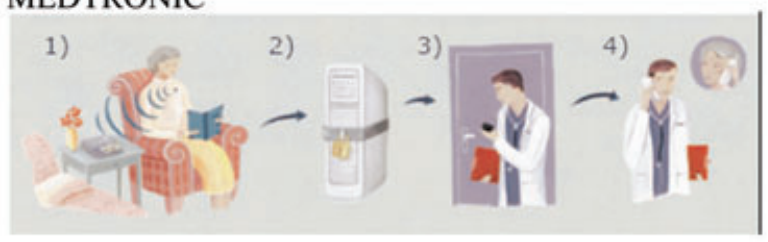

BOSTON Scientific

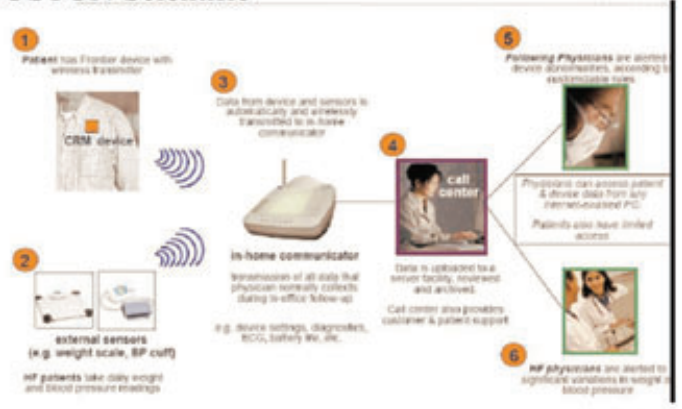

\section{SORIN GROUP}

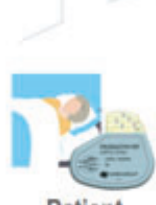

Patient

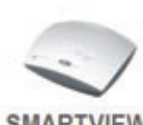

SMARTVIEW monitor

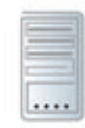

Network

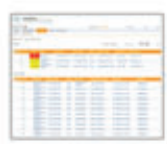

Web application

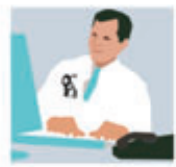

Physician \& helpdesk

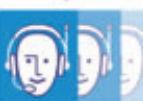

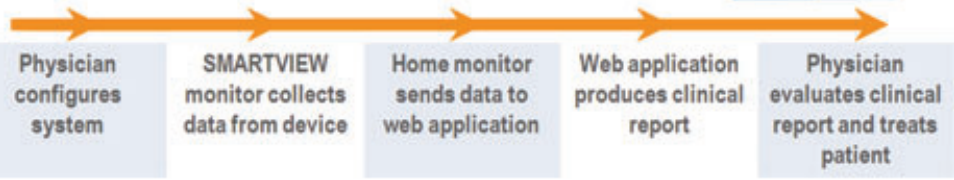

ST JUDE MEDICAL

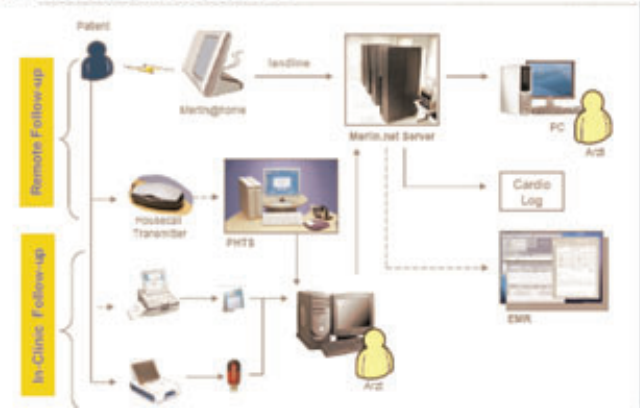

Figure 2. Different systems available for remote monitoring. Images reproduced with permission of the suppliers. 
managed. ${ }^{21,22}$ In the case of $\mathrm{AF}$, it is the fact that many recurrences of AF do not lead to symptoms but still represent a thread to the patient due to the risk of cerebral and systemic embolism which not only applies to patients on drug therapy but also to those after catheter ablation. The detection of asymptomatic AF influences further treatment strategies especially with regard to anticoagulation. ${ }^{23}$

Both patients with AF and those with syncope have to be monitored. The effectiveness of monitoring in detecting events depends on its continuity and duration. ${ }^{23-25}$

Optimal monitoring requires patients' acceptance, efficacy, simplicity of application, and costeffectiveness.

An important progress in this field at the end of the 20th century was the introduction of systems that monitor the electrocardiogram (ECG) with teletransmission of data (tele-event monitor with looping memory, and mobile cardiac outpatient telemetry [MCOT]). Such systems make it possible to assess parameters studied almost immediately without a patient having to visit a medical center. Tele-event Holters are limited to diagnosing symptomatic events since it needs to be activated by the patient who decides when and whether the recording should be initiated and data transmitted. Owing to the system of automatic analysis, MCOT enables both asymptomatic events and those reported by patients to be diagnosed. The adverse effects and the lack of acceptance by patients limit the duration of monitoring to a few weeks at most. Besides, it is difficult to make such monitoring continuously. ${ }^{26,27}$

This can be avoided by the use of ILRs, which have recently been called insertable cardiac monitors (ICMs). They are small devices, without any electrodes, which are implanted subcutaneously. Such features of these devices secure full comfort of an examination.

Most of the ICMs evaluated so far require visits to a hospital to have the recording analyzed. Since 2010, different devices have become clinically available. Current devices allow monitoring up to 3 years, to register at any time /during symptoms or when the patient or physician wishes so), to register automatically incidents of asystole, bradycardia, ventricular, and supraventricular tachycardia or $\mathrm{AF}$, and finally, to transmit data to the tele-center when the patient or the physician wishes so.
Although the implantation of the device is a small procedure and does not last longer than 20 minutes, it is not without complications (especially wound infections). Despite these potential complications inherent to any invasive procedure, an early use of ICMs appears to facilitate diagnosis of syncope patients. ${ }^{28}$

The Tele-ICM system may also be useful being considered in selected groups of AF patients, in diagnosing syncope, in selected groups with signs and symptoms suggestive of arrhythmia, in risk stratification in patients with depressed left ventricular function after acute myocardial infarction, and in patients with genetically inherited cardiac diseases, although formal clinical evaluation of the usefulness of ICM in most of these situations is lacking at present. ${ }^{29}$

\section{Atrial Fibrillation}

ICMs, like some PMs, have the capability to detect asymptomatic AF adequately, and with markedly higher sensitivity than intermittent ECG monitoring techniques. ${ }^{24-26,30}$

This has potentially relevant implications for the adequate diagnosis of $\mathrm{AF}$, e.g., in survivors of a stroke or a transient ischemic attack. Some preliminary data suggest that a high burden of AF may have implications for the likelihood of complications in AF patients, and ICMs are capable of measuring AF burden. ${ }^{31}$ ICM could help to assess the following questions: Whether anticoagulation therapy may be discontinued, ${ }^{32,33}$ to develop criteria of effectiveness of AF treatment, and to assess the real success rate of various therapies. AF treatment has increasingly involved catheter-based ablation, and a premium is placed on the accurate assessment of response for the determination of ablation results and patient care. Misclassification of a patient's response to the procedure can lead to serious errors in management, especially with regard to stroke prevention. It may be that an implantable loop recording system will provide the greatest degree of compliance, accuracy (sensitivity 95\% and specificity $85 \%$ ), and longevity for AF monitoring postablation, ${ }^{29}$ especially if systems can incorporate wireless and automated transfer of data (rather than device interrogation in an office). ${ }^{34}$

The clinical value of ICM after the diagnosis of $\mathrm{AF}$ has been established is relatively accepted in controlled trials, but is less obvious for routine clinical practice. 


\section{Syncope}

In 2009, the ESC in collaboration with EHRA, the Heart Failure Association of ESC, and the HRS released new guidelines on the diagnosis and management of syncope. ${ }^{22}$ They differ from the previous ones in that they lay emphasis on the increasing role of a diagnostic strategy based on prolonged monitoring (class 1 indication for an early usage in the diagnostic work-up). ${ }^{34,35}$

\section{Risk Stratification in Patients with Depressed Left Ventricular Function after Acute Myocardial Infarction}

Relatively common asymptomatic atrioventricular conduction disturbances, accompanied by impaired left ventricular contractile function, significantly increase the risk for sudden cardiac death. The experience from the Cardiac Arrhythmias and Risk Stratification after Acute Myocardial Infarction (CARISMA) study ${ }^{36}$ confirms the effectiveness of ILR in long-term assessment and ECG monitoring, providing that this group of patients should undergo further clinical evaluation.

\section{Risk Stratification in Genetically Inherited Cardiac Diseases}

Clinical observations suggest that prolonged ECG monitoring with the use of Tele-ICM could be applied in patients with such genetically conditioned diseases as Brugada syndrome, long QT syndrome or short QT syndrome, hypertrophic cardiomyopathy, or arrhythmogenic right ventricular cardiomyopathy. At present, TeleICM offers a chance to take reasonable decisions on further treatment especially in young people with asymptomatic congenital arrhythmogenic syndromes without documented dangerous arrhythmia.

\section{REVIEW OF EXISTING LITERATURE FOR TELEMONITORING OF DEVICES}

There are several papers supporting this technique, their usefulness, and benefits.

Lazarus et al. ${ }^{16}$ observed at the AWARE trial that the application of a monitoring system strongly supports its capability to improve the care of cardiac device recipients, enhance their safety, and to optimize the allocation of health resources.
He evaluated 3,004,763 transmissions made by 11,624 recipients of PMs $(\mathrm{n}=4631)$, defibrillators (ICDs; $\mathrm{n}=6548)$, and CRT-D systems ( $\mathrm{n}=445)$ worldwide. The duration of monitoring/patient ranged from 1 to 49 months, representing 10.057 years. The vast majority (86\%) of events were disease-related. The mean interval between the last follow-up and the occurrence of events notified by HM was 26 days, representing a putative temporal gain of 154 and 64 days in patients usually followed up at 6- and 3-month intervals, respectively.

Lead dysfunctions in ICD patients can lead to inappropriate shocks and Spencker et al. ${ }^{37}$ evaluated how HM systems help physicians to react quickly on these serious ICD malfunctions. He evaluated 54 patients who had to undergo resurgery due to malfunctions of the ICD lead. Eleven of them were on HM interrogating the device on a daily basis. The rate of inappropriate shocks and symptomatic PM inhibition due to oversensing was compared with the 43 patients without remote surveillance. Home monitoring sent alert messages in $91 \%$ of all incidents. Eighty percent of the patients were asymptomatic at the first onset of oversensing. Compared with the patients without HM, inappropriate shocks occurred in $27.3 \%$ in the HM group versus $46.5 \%$ ( $\mathrm{P}=$ n.s.). Event messages were dispatched in a mean of 54 days after the last ICD interrogation and 56 days before the next scheduled visit. Thus, 56 days of reaction time are gained to avoid adverse events. The authors conclude that the diagnosis could be established correctly by an alert of the HM system, and it might have a potential to avoid inappropriate shocks due to lead failure and $\mathrm{T}$-wave oversensing. ${ }^{37}$

Heidbuchel et al..$^{38}$ analyzed the significant burden on specialized electrophysiology clinics which the follow-up of ICDs poses due to the increased number of device implantations with regular inoffice visits every 3-6 months.

They evaluated 1739 prospectively coded ICD visits in a random set of 169 patients. The standard follow-up scheme consisted of in-office visits 1 month after implantation, and then every 6 months, unless approaching battery depletion. The proportion of relevant findings during unscheduled visits was significantly higher than during scheduled visits $(80.6$ vs. $21.8 \% ; \mathrm{P}<0.0001)$ and a higher proportion of those was arrhythmiaand/or device-related (85.1 vs. $55.3 \%$; $\mathrm{P}<0.0001$ ). Reprogramming was required more often (33.1 
vs. $4 \% ; \mathrm{P}<0.0001)$ and hospitalization rate was higher (18.3 vs. $2 \% ; \mathrm{P}<0.0001)$ so that $51.4 \%$ of unscheduled visits would require in-office evaluation. Overall, remote follow-ups would correctly exclude device function abnormalities or arrhythmic problems in $1402(82.2 \%)$ cases, identify an arrhythmic problem in $262(15.3 \%)$, and correctly identify a device-related problem in 35 (2.1\%). Clinical evaluation would diagnose a relevant clinical problem without any device interrogation abnormality in 170 patients $(10 \%)$. They conclude that ICD RM can potentially diagnose $99.5 \%$ of arrhythmia-or device-related problems if combined with a follow-up by the local general practitioner and/or referring cardiologist. Its use may provide a way to significantly reduce in-office follow-up visits that are a burden for both hospitals and patients.

A similar study was performed by Elsner et al. [REFORM]. ${ }^{39}$ They investigated in a prospective, randomized, and multicenter comparison study the effect of ICD HM against conventional follow-up (FU) in 115 MADIT II patients. The results prove that the simplified ICD follow-up scheme with additional HM in MADIT II patients can reduce the number of visits and lead to time reduction.

In a very interesting review, Schmidt et al. ${ }^{40}$ evaluated how telemonitoring can improve the medical care, quality of life, and prognosis on patients with chronic congestive heart failure. They searched the Medline database for articles appearing from June 2001 to May 2008, with an emphasis on randomized, controlled trials. Their data suggested that telemonitoring is effective, yet there is no evidence for superior outcomes with any particular model of care incorporating telemonitoring (i.e., monitoring of vital signs vs. structured telephone monitoring).

The PREFER study ${ }^{10}$ was a prospective, randomized, parallel, unblinded, and multicenter trial to determine the utility of remote PM interrogation for the earlier diagnosis of clinical events compared with the existing practice of TTM and routine office visits. There were 866 clinical events reported in 382 patients in the study. The number of events reported per patient was 0.517 in the remote arm and 0.308 in the TTM, and the most frequent was nonsustained $\mathrm{VT}$, followed by atrial tachycardia/AF episodes lasting 48 hours or longer. The authors conclude that the use of remote PM interrogation follow-up detects clinically important events that require action more quickly and frequently than transtelephonic rhythm strip recordings. Similar results were observed at the CONNECT study. ${ }^{41}$

Jung et al. ${ }^{5}$ published data collected in several completed and ongoing studies strongly suggest that this new technology will make important contributions, particularly with respect to the facilitation of ICD follow-ups, enhancement of patient safety, and quality of life. The life-saving potential of automatic daily RM messages has been illustrated by reports of lead failures and device dysfunctions: Neuzil et al. ${ }^{42}$ described the failure of an ICD to properly charge its capacitors and deliver appropriate therapy. Spencker et al. ${ }^{43}$ observed a fall in atrial pacing impedance from daily remote messages. Ritter and Bauer ${ }^{44}$ reported two cases of inappropriate VT detection caused by supraventricular tachycardia and intermittent T-wave oversensing, respectively, both diagnosed on the basis of remote transmissions of intracardiac electrogram, and immediately managed at the time of nonroutine follow-ups. These cases, observed during the early years of HM, will likely increase in numbers as its clinical applications increase.

\section{INDICATIONS}

\section{Diagnosis of Symptomatic and Asymptomatic Events}

Electronic cardiovascular implantable devices collect, quantify, and analyze important information regarding their own function and patient conditions. These data are conventionally accessed by physically downloading stored diagnostic information. Monitoring guidelines recommend a followup method based on frequent in-person scheduled evaluations. ${ }^{4}$ This ignores problems occurring between calendar-based scheduled appointments in the majority of the time. Remote monitoring may solve this problem; however, technologies differ in application. Earlier systems demanded patientactivated transmissions on a calendar basis. Short, small-scale feasibility studies demonstrated the ability to detect asymptomatic lead and generator problems, e.g., T-wave oversensing, battery elective replacement indicators (ERI). ${ }^{8,17}$ When used to follow up a PM population, only $66 \%$ of data were transmitted and clinical events requiring action were detected several months later. ${ }^{10}$

Automatic RM, in contrast, promises the maintenance of near-continuous surveillance 
independent of schedule or patient or physician interaction. Home monitoring self-tests system performance daily and employs automatic device-triggered transmissions for rapid problem notification regardless of interrogation schedules. ${ }^{45}$ These may concern arrhythmias, device activities, or acute deviations from established trends (e.g., $\mathrm{AF}, \mathrm{VF}, \mathrm{ERI}$, impedances out of range). ${ }^{45,46}$ Events may be transmitted immediately and flagged for attention irrespective of associated symptoms. This is especially important for potentially dangerous silent events.

Automatic RM was prospectively tested and compared with conventional in-person follow-up in the Lumos-T Safely RedUceS Routine Office Device Follow-up (TRUST) multicenter trial. ${ }^{47,48}$ Early detection was assessed by time elapsed from event onset to physician evaluation. In conventional care, this occurred at in-office interrogation (scheduled or unscheduled). Evaluation in HM occurred on receipt of event notifications in response to detection of preprogrammed events or in-office interrogation (scheduled or unscheduled). Investigators recorded whether these events were clinically asymptomatic (silent). TRUST results demonstrated that HM enhanced problem discovery of clinically silent as well as symptomatic events, despite less frequent hospital evaluations. ${ }^{48}$ Detection was advanced by more than 30 days compared with conventional care and this advantage would be greater if conventional visits were scheduled 6 or 12 monthly. Arrhythmias were the most common cause for event notifications. Median time from onset to physician evaluation of combined first AF, VT, and VF events in $\mathrm{HM}$ was 1 day, significantly less than the value in conventional care of 35.5 days (Fig. 3).

System-related problems occurred infrequently but were often asymptomatic. Conventional follow-up resulted in delayed detection and underreporting of important events. ${ }^{48}$ In contrast, HM enabled prompt detection (less than 24 hours). Event triggers covered an extensive range of potentially lethal (and asymptomatic) system problems, e.g., ERI, lead fracture, high-voltage circuitry failure, and permit prompt intervention either surgically, e.g., for lead failure, ${ }^{49-51}$ or conservatively, e.g., to prevent potential inappropriate therapies (e.g., electromagnetic interference, AF). The nonsustained ventricular arrhythmia notification may be triggered by system issues such as lead electrical noise artifacts caused by fracture or nonphysiological electrical signals. Identification of patients with

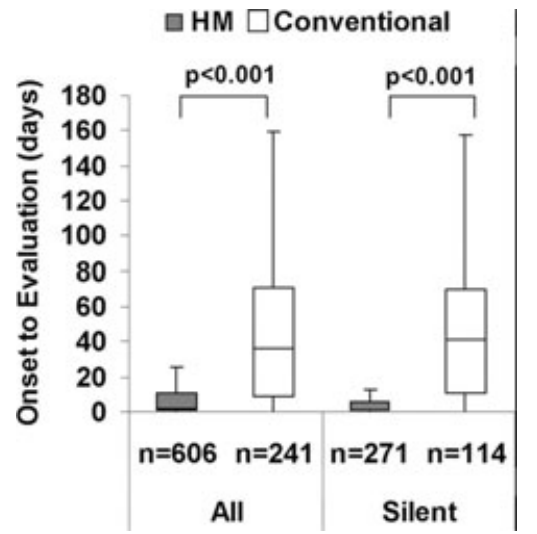

Figure 3. Legend HM versus conventional care [days]: AF: median, 5.5 versus 40; interquartile range, 1-51.25 versus 15.5-59; VT: median, 1 versus 28; interquartile range, 1-6 versus 6.5-69.25; VF: median, 1 versus 36; interquartile range, 1-7 versus 10-75; SVT: median, 2 versus 39; interquartile range, $1-19.5$ versus 8.569. Clinically asymptomatic(silent) problems were also detected early in $\mathrm{HM}$ for combined first AF, VT, VF, or SVT events (median, 1 vs. 41.5; interquartile range, 1-6 vs. 10.5-70.25). Compiled with permission from Varma et al. ${ }^{48}$

a high burden of these may facilitate intervention to preempt premature battery depletion. ${ }^{52}$ Notification for disabled VF detection is important as more patients with different comorbidities undergo procedures in different departments.

These data illustrate that problems occurring in patients with implanted devices are often clinically silent but may be revealed by automatic RM technology. The ability to generate parameter trends with high temporal resolution permits adjudication of asymptomatic deviations from baseline values. Plots that are updated frequently when coupled with ability for rapid notification deviations may enable therapeutic intervention. ${ }^{53}$

\section{Utility in Certain Pathologies: Congestive Heart Failure and Atrial Fibrillation}

Heart Failure

There are several methods currently available to identify patients at risk of worsening heart failure (Table 3). Commercially available methods include assessment of weight as well as intrathoracic impedance. The feasibility of daily monitoring of weight (with the physician alerted to a weight gain or loss of 2 pounds over 2 days or 5 pounds over 
Table 3. Device Parameters Used to Track the Heart Failure Patient

"Vital signs"
Weight and blood pressure (daily)
Symptoms
Quality of life questions (weekly)
Assessment of patient activity
Lead-related
Significant increase in pacing thresholds,
especially the left ventricular lead
Significant increase in the percentage of right
ventricular pacing
Significant decrease in the percentage of left
ventricular pacing
Arrhythmia-related
Atrial tachyarrhythmias
Ventricular tachyarrhythmias
Miscellaneous
Intrathoracic impedance
Heart rate variability
Respiratory rate

a week) has recently been demonstrated in a large clinical trial; ${ }^{54}$ however, long-term outcome data are still needed. Intrathoracic impedance appears to be inversely correlated with pulmonary capillary wedge pressure and fluid balance; it declines before the onset of patient symptoms and before hospital admission for fluid overload. ${ }^{5,56}$ However, as a singular indicator, it lacks adequate sensitivity and specificity to guide clinical decision making. More recently, a system to measure left atrial pressure directly has been reported; ${ }^{57}$ a large-scale trial to evaluate the system clinically is currently underway.

Abraham et al. $^{58}$ evaluated patients with New York Heart Association (NYHA) Class III heart failure who were randomly assigned by use of a wireless implantable hemodynamic monitoring system or to a control group for at least 6 months. Eighty-three heart-failure-related hospitalizations were reported in the treatment group (n = 270) compared with 120 in the control group ( $\mathrm{n}=280, \mathrm{P}=0.0001)$. During the entire followup, the treatment group had a 39\% reduction in heart-failure-related hospitalization compared with the control group (153 vs. 253; $\mathrm{P}=0.0001$ ). These results are consistent with, and extend, previous findings by definitively showing a significant and large reduction in hospitalization for patients with NYHA Class III heart failure who were managed with a wireless implantable hemodynamic monitoring system.

\section{Atrial Fibrillation}

AF is the most commonly encountered arrhythmia in clinical practice, is a major cause of ischemic stroke, and can contribute to the development of heart failure. For all these reasons, confirming the diagnosis of $\mathrm{AF}$ is important in many patients, and defining response to therapeutic interventions is crucial irrespective of treatment option. There are several reasons that establishing the presence and quantity of $\mathrm{AF}$ has been challenging: $\mathrm{AF}$ can have a multitude of symptoms, but none are specific for diagnosis of this arrhythmia; many patients have asymptomatic AF episodes even when the patient is known to suffer from symptomatic AF; and therapeutic interventions (both drug and ablation) may alter the perception of AF. Hence, ECG confirmation is considered the gold standard for AF detection. Historically, the 12-lead ECG and ambulatory monitoring were the common modes used for AF recording, but these modalities are too brief to provide assurance of detection of $\mathrm{AF}$ and its pattern or its absence.

Prolonged or permanent arrhythmia monitoring is generally restricted to permanently installed devices including PMs, defibrillators, and resynchronization systems, provided that an atrial lead has been implanted. Continuous atrial sensing and programmable algorithms that detect elevated atrial rates are the primary means by which atrial tachyarrhythmias can be captured and quantified with high sensitivity and good specificity. It is important to recognize that a detected atrial high rate event (AHRE) is not synonymous with AF and is sensitive to the programmed rate cutoff and duration, and is further limited because only a single atrial site is sampled. There may also be oversensing on the atrial channel and thus diminished specificity. Acquired data can be interrogated in a clinic or obtained through RM and can include the atrial rate and regularity, associated ventricular rate, electrographic confirmation, duration of episode(s), and frequency of episodes. The latter two may be utilized to calculate what is commonly referred to as "AF burden." One study demonstrated the utility of newly implanted PMs for the initial diagnosis of $\mathrm{AF}$ with an incidence of about $25 \%$ over 1 year. ${ }^{59}$

The clinical significance of AHREs is still being debated. In a provocative analysis, the MOST investigators concluded that the presence of $\geq 1$ AHRE of $\geq 5$ minutes at 1 year was associated with a slightly higher mortality rate although not 
a higher stroke rate. ${ }^{60}$ These brief PM-detected events may simply be a marker of older age, more advanced cardiovascular disease, and prior AF. Indeed, the duration of AF detection from implanted PMs has been correlated to the risk of stroke, greatest when AF exceeded 24 hours, with risk further modulated by conventional criteria. ${ }^{33}$

The threshold at which AHRE detection should trigger intervention including anticoagulation in higher risk patients has not been defined. In a recent investigation, ${ }^{61}$ only a borderline increased risk of stroke was calculated in a convoluted secondary analysis when the AHRE burden exceeded 5.5 hours compared with no or shorter duration AHRE, but the overall event rate was so low that the primary endpoint comparisons could not be performed. Several important questions still need to be addressed including whether any single episode longer than a prespecified duration is critical, or whether the overall burden of arrhythmia is more important.

As contemporary devices also have the capability of RM and physician notification based on prescribed criteria, they can serve as early-warning systems should an arrhythmic event be detected. ${ }^{18}$ In the TRUST study, ${ }^{48}$ the time to physician evaluation for a detected AF event was reduced from 40 to 6 days. Whether this leads to a favorable clinical outcome was not tested. This technology, however, could be exploited to allow targeted anticoagulation (or antiarrhythmic therapy) if and when AF is detected in an early phase (e.g. at 12-48 hours after onset), and even anticoagulation withdrawal when AF has subsided. Devices could be programmed to alert the patient directly or could be monitored and verified by a centralized service provider. This unproven, but tantalizing, tailored approach is being tested in a randomized clinical trial versus standard care. ${ }^{62}$ The recent availability of the rapidly acting oral anticoagulant dabigatran (as opposed to warfarin) makes this strategy particularly attractive. Hanke et al. evaluated 45 cardiac surgical patients treated with either left atrial epicardial high-intensity focus ultrasound ablation $(\mathrm{n}=33)$ or endocardial cryothermy $(\mathrm{n}=12)$ in the case of concomitant mitral valve surgery. ${ }^{63} \mathrm{Si}$ nus rhythm was documented in 53 readings of 24 Holter Monitoring, in 34 of these instances by the implantable continuous cardiac rhythm monitoring in the time period before $24 \mathrm{HM}$ readings $164 \%$; $\mathrm{P}=$ $0.0001)$, reflecting a $24 \mathrm{HM}$ sensitivity of 0.60 and a negative predictive value of 0.64 for detecting $\mathrm{AF}$ recurrence. The authors concluded that continuous heart rhythm surveillance instead of any conventional 24HM follow-up strategy is necessary in this group of patients.

\section{BENEFITS OF REMOTE MONITORING}

There are many potential benefits of remote patient monitoring (RPM) for the patient and their caregivers, the follow-up centers, the health care infrastructure, the manufacturer, and the discipline of cardiac implantable electronic device (CIEDs) management. ${ }^{4}$

Several recent studies /COMPAS, CONNECT, OEDIPE, PREFER, REFORM, and TRUST $^{10,13,39,41,48,64,65}$ ) in addition to important registry data (ALTITUDE ${ }^{13}$ ) today constitute a strong evidence base for RM. In the very early days of RM, there was concern expressed, as there is with most new technologies, that patients and caregivers would not be satisfied with RM because they would lose the advantage of a face-to-face visit, despite the fact that scheduled, routine follow-up visits have, in practice, a very low actionability rate. ${ }^{48}$ Today multiple studies have shown that both patients and caregivers are satisfied with RM both in terms of its ease of use and continued connectedness to the follow-up center. ${ }^{66}$

In addition, RM is adding strong benefits to the patient's peace of mind, psychological well-being, and safety specifically following an advisory and therefore is seen as an important alternative to the current standard of care. ${ }^{43,67-69}$

In addition, patients require fewer overall visits to the follow-up clinic when RM is used. Both very large trials, the CONNECT ${ }^{41}$ and TRUST, ${ }^{48}$ demonstrated a significant cut in the total number of in-office visits (3.92 in RM and 6.27 in-office in CONNECT and 2.1 in RM and 3.8 in-office in TRUST). Further trials and real-life studies showed a cut in in-office visits by up to $63 \% .{ }^{39,70}$ This significant reduction contributes to the effectiveness of the health care system as the workload for caregivers in the device clinic is reduced by RM. ${ }^{39}$ In the study by Raatikainen et al., ${ }^{71}$ physicians and nurses' time required for an office visit versus a follow-up by RM was assessed. The physician time required to review the RM data $(8.4+$ 4.5 minutes, range 2-30 minutes) was significantly shorter than the time needed to complete a device 
follow-up visit in the clinic $(25.8+17.0$ minutes, range 5-90 minutes), $\mathrm{P}=0.001$. Likewise, allied professionals spent more time on an in-clinic visit when compared with an RM follow-up assessment $(45.3+30.6$ vs. $9.3+15.9$ minutes; $P=0.001)$.

The significant higher adherence rate of RM versus Control (RM 92.7\% vs. Control 89.2\%, P = $0.001)$ may be explained by the lower burden for the patient, the caregivers, and the dramatically higher actionability rate of an RM-triggered follow-up compared with a scheduled follow-up visit. $^{48}$

\section{Remote Monitoring Provides Timely Detection of Clinical Events}

Another important benefit is that RM detects clinical abnormalities that would be either completely missed by less frequent in-office visits, or detected significantly later in the absence of close to continuous RM data assessment. Clinical data demonstrate earlier detection of clinical events of up to 148 days. $^{10,37,41,48,61}$ The TRUST trial ${ }^{48}$ reports that median time from onset to physician evaluation of combined first AF, VT, and VF events was significantly reduced from 35.5 days to only 1 day in the remote follow-up arm. Furthermore, even though very rare problems with pulse generators and leads were detected significantly earlier (RM $4.4+9.2$ days vs. Control $23.6+40.2$ days) ${ }^{48}$ Overall, the study detected 20 device-related problems that required surgical revision; 15 of these were detected by RM and only 5 in their control group. The CONNECT trial ${ }^{41}$ reports that the time from a clinically significant event to making a clinical decision in the RM arm was 4.6 days in the remote arm versus 22 days in the in office arm significantly shorter. Perhaps, as a result of earlier detection of clinical problems, resulting hospitalizations may be shorter in those patients on RM. In the CONNECT trial, ${ }^{41}$ the RM arm of the trial had significantly shorter hospitalization length of stay than those patients followed in the clinic (3.3 vs. 4.0 days; $P=0.002$ ). A broad benefit of $\mathrm{RM}$ is the ability to obtain clinically useful data from very large-scale registries offering an insight into real-world outcomes. For example, observational data of the large-scale ALTITUDE study ${ }^{13}$ demonstrated at 1 and 5 years higher survival rates for ICD/ CRT-D patients on RM versus the standard of care group (50\% reduction, $\mathrm{P}=0.0001$ ). However, the lack of clinical profile data and specific knowledge of comorbid conditions in this registry limits interpretation and assignation of clinical significance to this novel observation and supports future studies aimed at confirming this observation. Other observational studies demonstrated improved clinical management of patients with CIED. ${ }^{18}$ There is a clear need to assess whether earlier detection of events results in better management and outcome of patients on RM, or whether earlier detection of events increases health care utilization to an extent that offsets these potential benefits.

\section{LEGAL CONSIDERATIONS}

The rapid evolution and growing use of RM will likely present new legal challenges. The transmission, storage, sharing, and interpretation of CIED diagnostics each will fall under scrutiny to assure that patients' and health care providers' rights are maximally protected. In the USA, the Health Insurance Portability and Accountability Act (HIPAA) of 1996, the Health Information Technology for Economic and Clinical Health Act, and Code of Federal Regulations provide a general framework addressing the security and privacy of Protected Health Information. Health care providers and health care organizations that are involved in RM of CIEDs will typically sign a "Terms of Use" agreement and when applicable a "Business Associate Agreement" with each of the CIED vendors. These legal documents outline the provisions of RM between the CIED vendor and the user.

The patient needs to be informed the purpose and limitations of RM, such as the fact that it does not replace an emergency service or absence of dealing with alert events outside office hours. Before initiating RM and follow-up, the patient may be requested to sign a written informed consent stating these points and authorizing transmission of personal data to third parties, respect of privacy, and confidentiality of patient data by device companies should be subjected to strict rules, described in contracts.

Vulnerability of security breaches by hackers accessing devices with wireless capability must be tested in every system. Halperin et al. ${ }^{72}$ performed laboratory tests using several software radio-based attacks that were able to retrieve encrypted personal patient data, as well as to reprogram device settings (including commanded shocks). This report triggered considerable media coverage, 
although it is believed that the risk of unauthorized access to an ICD is unlikely, given the considerable technical expertise required. ${ }^{73}$ There have been no reports to date of unauthorized reprogramming of implantable devices; however, unauthorized access to personal information stored on Internet servers must also be considered.

Cardiac implantable devices record a wealth of information and as devices become more sophisticated, the scope of information can be expected to grow. Current CIEDs provide not only arrhythmia information but also several indicators of heart failure.

Cardiac implantable device transmissions may occur either over telephone lines or over cellular network lines. These transmissions often only take less than a minute to a few minutes to complete. However, in the foreseeable future, we can expect alternative methods of data transmission to become available with transmission rates that will make it possible for nearly continuous and instantaneous patient CIED data delivered to health care providers. There are, of course, limitations to how frequently CIED data can be reviewed by health care providers and battery longevity constraints will likely limit the transmission times as well. If, for example, an ICD lead alert suggestive of an impending lead failure becomes available on a Saturday at midnight, what would be considered a "reasonable" response time to this alert? What if that night the same patient developed ventricular fibrillation and ICD therapies failed, what would have been a "reasonable" response time then? Institutional guidelines and/or caregiver and patient contracts may need to be devised so as to limit the periods of liability. In addition, guidelines may need to be established to determine the periodicity with which CIED transmissions would need to be reviewed and documented.

The growing number of patients with CIEDs and the accumulating data may lend itself to vital analyses and may yield significant prognostic information. However, access to these data is another area that will require legal inquiry. Who should be the custodian of these data, if anyone? Who should be able to access these data, if anyone? What may be the effect of any results on a class of patients?

Technological advancements continue to structure our practice of medicine, but with it, often new legal challenges emerge. In order to minimize risk to patient and liability to health care providers, a clear discussion regarding the expectations and limitations of RM between patients and health care providers is recommended.

\section{REIMBURSEMENT CONSIDERATIONS}

Despite the value of CIEDs today, there remains considerable need for follow-up therapy improvement to maximize patients' health and safety. Continuous patient and device RM and the replacement of often ineffective ${ }^{48}$ but resourceintensive calendar-based follow-ups ${ }^{4,5}$ would contribute strongly to the effective prevention, detection, and sufficient management of medical or technical events. The significant limitations of today's in-office follow-up such as late detection of medical or technical events with potentially serious health outcome implications and the high resource intensity of calendar-based follow-up often leading to adherence challenges have been described in more detail elsewhere. ${ }^{6,10,41,74,75}$ Remote monitoring has been developed to address these limitations by offering need-based and continuous surveillance of the devices and patients to improve safety and cost-effective delivery of health care.

Today, physician reimbursement remains a major concern with a lack of appropriate reimbursement in place in most countries worldwide and as a result limiting an increased use of evidence-based RM.

Today's cost containment pressure requires increased reimbursement efforts with the burden of proof shifting to medical communities and manufacturers. Reimbursement assessments often begin with the presumption that a technology or service will not be covered unless its use is supported by scientific evidence of improved outcomes. ${ }^{74}$ Whereas it is generally out of question that physicians need to be adequately paid for their services, the current discussions around physician reimbursement are focused on the questions whether $\mathrm{RM}$ is safe, effective, and cost-effective. ${ }^{76-78}$ However, existing health technology assessments are at least partially obsolete as they could not take the only recently available level 1 evidence into consideration at the time of their review ${ }^{74-76,79}$ (Table 4).

In 2010/2011, on top of observational data of more than 20,000 patients, the scientific evidence base for RM has significantly changed with the publication of the results of five RCTs with altogether more than 4000 patients. ${ }^{10,20,29,39,41,48,65,71}$ 
Table 4. Reimbursement for Remote Monitoring of Cardiac Devices

\begin{tabular}{|c|c|c|c|c|}
\hline \multirow[b]{2}{*}{ Country/Region } & \multirow[b]{2}{*}{ None } & \multicolumn{2}{|c|}{$\begin{array}{l}\text { Hardware and Industry } \\
\text { Service Reimbursement }\end{array}$} & \multirow{2}{*}{$\begin{array}{c}\text { Physician } \\
\text { Reimbursement } \\
\text { Category } 3 \\
\text { Remote } \\
\text { Calendar } \\
\text { based FU }\end{array}$} \\
\hline & & $\begin{array}{c}\text { Category } 1 \\
\text { RF-enabled } \\
\text { implantable } \\
\text { device premium }\end{array}$ & $\begin{array}{c}\text { Category } 2 \\
\text { Patient Monitor, } \\
\text { Communication } \\
\text { Service }\end{array}$ & \\
\hline Africa & $\mathrm{X}$ & & & \\
\hline Asia-Pacific/Australia & $\mathrm{X}$ & & & \\
\hline Baltic states & $\mathrm{X}$ & & & \\
\hline Belgium & $\mathrm{x}$ & & & \\
\hline Canada & $(x)$ & & & $\begin{array}{l}X \text { (Province of } \\
\text { Alberta only) }\end{array}$ \\
\hline Czech Republic & & & & $\mathrm{x}$ \\
\hline Central America & $\mathrm{X}$ & & & \\
\hline Denmark & $\mathrm{x}$ & & & \\
\hline Finland & & & & $\mathrm{x}$ \\
\hline France & & $\begin{array}{l}\text { X (BIOTRONIK and } \\
\text { Boston Scientific } \\
\text { only) }\end{array}$ & & $x$ \\
\hline Germany & & & $\begin{array}{l}\text { X (individual insurance } \\
\text { contract, BIOTRONIK only) }\end{array}$ & $x$ \\
\hline Italy & $\mathrm{x}$ & & & \\
\hline $\begin{array}{l}\text { Japan } \\
\text { Netherlands }\end{array}$ & & $x$ & & X (with restrictions) \\
\hline $\begin{array}{l}\text { Portugal } \\
\text { Portas }\end{array}$ & & & & $\begin{array}{c}x \text { (for IPG only) } \\
x\end{array}$ \\
\hline Russia & $\mathrm{X}$ & & & \\
\hline South-Eastern Europe & $\hat{x}$ & & & \\
\hline Southern America & $\mathrm{X}$ & & & \\
\hline Sweden & & & & $\mathrm{x}$ \\
\hline Swizerland & $\mathrm{x}$ & & & \\
\hline UK & $\mathrm{x}$ & & & \\
\hline USA & & & & $\mathrm{x}$ \\
\hline
\end{tabular}

The table presents the current reimbursement status for remote monitoring of CIEDs. Three reimbursement categories can be differentiated. Reimbursement needs to be established for the time spends by the physician to assess the information (category 3). In addition, reimbursement tariffs need to be established for the transmitting hardware and the transmission costs such as telephone costs (category 2) as well as for the RF-enabling CIEDs (category).

Reimbursement categories

1. Countries with higher reimbursement for selected RF-enabled implantable devices.

2. Countries with reimbursement for patient monitors or with approved reimbursement codes for remote follow-up or implanted cardiac devices for doctors/clinics.

3. Countries where existing codes for in-office follow-up are being used to reimburse for remote follow-up for doctors/clinics. From Eucomed (CRM Telemonitoring Working Group). Business development (2009).

A strong safety profile, the effectiveness, and a positive impact on resource utilizations in comparison with calendar-based in-office follow-up visits have been consistently and repeatedly demonstrated. ${ }^{32,34,36,37,41}$

The vast majority of ICD recipients have some degree of left ventricular systolic dysfunction and these patients are at risk of heart failure hospitalizations, which impose a major economic burden on the health care system. So, concerned were the Centers for Medicare and Medicaid Services about the adverse economic implications in the United States of recurrent heart failure hospitalizations that, in January 2009, they afforded favorable financial reimbursement to physicians who chose to manage device patients remotely. Specifically, monthly reimbursement is available for RM of an "implantable cardiovascular monitor system;" the latter, which can be either a stand-alone device or incorporated into a PM or ICD system, provides physiologic cardiovascular data elements from internal (transthoracic impedance, heart rate 
variability, respiratory rate, and intracardiac pressures) and external (weight and blood pressure) sensors. A recent meta-analysis suggests a reduction in hospitalizations and death in heart failure patients monitored remotely as compared with usual care. ${ }^{79}$

A recent meta-analysis by Klersy et al. ${ }^{80}$ showed that management of heart failure patients by RM is cost-saving due to a substantial reduction in health care resource utilization mostly driven by a reduction in the number of HF hospitalizations. The cost saving expected in both European and US health care systems is linearly related to the implementation rate of RPM. An important caveat is the limited follow-up time of the studies considered in this meta-analysis, which restricted the time horizon for the cost-effectiveness assessment to 1 year. The novel cost-effectiveness data coupled with the demonstrated clinical efficacy of RPM should encourage its acceptance among clinicians and its consideration by third-party payers. At the same time, the scientific community should acknowledge the lack of prospectively and uniformly collected economic data and should request that future studies incorporate economic analyses. However, one should recognize that most of the cost-effectiveness data analyses are frequently considering relatively outdated technologies or management disease programmes, which have not included implantable device based; thus, extrapolation to device-based telemonitoring should be done cautiously. ${ }^{81}$

With this supportive evidence in place, the responsibility for establishing reimbursement policies is now shifting back to the policy makers. Today's evidence demonstrates that a replacement of calendar-based follow-ups with RM can increase patient safety by early detection of technical events, reduce the number of in-office follow-ups, detect medical events early, may reduce length of stay and hospitalization rates, may reduce the risk of stroke and atrial arrhythmias, and may cut down mortality risk by about $50 \%$. 7,10,13,41,48,60,66,76

The lack of appropriate reimbursement led to a relative paucity of real-life outcomes research data. Therefore, some, however, manageable reallife performance uncertainty of RM may remain. Reimbursement policies for RM of CIEDs services and hardware need to be enforced in a timely manner to remove a remaining barrier for a more widespread use of evidence-based RM of CIEDs to significantly improve postimplant patient management. Innovative reimbursement schemes such as coverage with evidence in development ${ }^{60}$ might be a viable option to overcome the current discrimination of RM reimbursement. Based on today's convincing evidence in place, the utilization of RM should not be further limited by discriminative reimbursement policies but should be left to the decision making of doctors and patients to optimize individual patient care.

\section{FINAL COMMENTS}

Telemedicine is a rapidly growing area where technological developments currently far exceed clinical experience and evidence-based strategies. Remote monitoring of CIEDs represents a growing market with increasing numbers of patients being subject to these technologies, but also more and more physicians are involved in decision making on the indications for these technologies and the handling of data in the context of clinical decision making.

As device technology is fast moving and the operational mode for performing telemonitoring is rapidly evolving, it is important to consider that novel operational platforms for in- and outof-hospital patient management are highly needed, new education and competence definition of allied professionals are needed, and urgently require attention from scientific societies. Finally, given the significant economical burden that telemedicine may pose on an already very fragile and underfinanced health care system, it is imperative to find the appropriate timing cycle for economical evaluation of novel technologies and for fast update of health technology assessment.

Two international organizations agreed upon this consensus statement which has assembled the currently available information on systems and on results of their use under various clinical conditions.

Future research in this area will need to concentrate on several issues of which a few will be mentioned here:

(1) What is the sensitivity and specificity of data obtained from CIEDs under various specific clinical conditions?

(2) What is the time interval between CIEDbased detection of an abnormality and measures to be taken by the responsible physician or the patient?

(3) While RM is intended to reduce the need for some face-to-face scheduled clinic visits, what 
is its impact on various outcome parameters such as quality-of-life, adverse events, even on mortality, etc.?

(4) With increasing number of patients with CIEDs, what is the impact on physician and technician working load?

(5) What is the cost-effectiveness ratio of such systems?

(6) What are potential, not yet identified problems with regard to patient data protection, and what are the potential differences of these problems with various national and international legal systems (with data stored outside one's own country)?

These and other questions will have to be answered by further research in this area. If this is encouraged by this document, then a major aim has been reached besides presenting the currently available knowledge and experience of the use of CIEDs.

\section{REFERENCES}

1. Hayes DL, Naccarelli GV, Furman S, et al. North American Society of Pacing and Electrophysiology. NASPE training requirements for cardiac implantable electronic devices: Selection, implantation, and follow-up. Pacing Clin Electrophysiol 2003:26(Pt1):1556-1562.

2. Nacarelli GV, Conti JB, DiMarco JP, et al. Task Force 6: Training in specialized electrophysiology, cardiac pacing, and arrhythmia management. Heart Rhythm 2008;5:332337.

3. Eucomed http://www.eucomed.org/uploads/_medical_ technology/facts_figures/110518_statistics_for_cardiac_ rhythm_management_products_20052010.pdf

4. Wilkoff BL, Auricchio A, Brugada J, et al. HRS/EHRA expert consensus on the monitoring of cardiovascular implantable electronic devices (CIEDs). Europace 2008;10:707-725.

5. Jung W, Rillig A, Birkemeyer R, et al. Advances in remote monitoring of implantable pacemakers, cardioverter defibrillators and cardiac resynchronization therapy systems. J Interv Card Electrophysiol 2008;23:73-85.

6. Burri H, Senouf D. Remote monitoring and follow-up of pacemakers and implantable cardioverter defibrillators. Europace 2009;11:701-709.

7. Winters SL, Packer DL, Marchlinski FE, et al. North American Society of Electrophysiology and Pacing. Consensus statement on indications, guidelines for use, and recommendations for follow-up of implantable cardioverterdefibrillators. Pacing Clin Electrophysiol 2001;24:262-269.

8. Schoenfeld $M H$, Reynolds DW. Sophisticated remote implantable cardioverter-defibrillator follow-up: A status report. Pacing Clin Electrophysiol 2005;28:235-240.

9. Schoenfeld MH, Compton SJ, Mead RH, et al. Remote monitoring of implantable cardioverter defibrillators: A prospective analysis. Pacing Clin Electrophysiol 2004;27(Pt I): 757-763.

10. Crossley GH, Chen J, Choucair W, et al., on behalf of the PREFER study investigators. Clinical benefits of remote versus transtelephonic monitoring of implanted pacemak ers. J Am Coll Cardiol 2009;54:2012-2019.

11. Thenus Da, Res JC, Jordaens LJ. Home monitoring in ICD therapy: Future perspectives. Europace 2003;5:139-142.

12. Stellbrink C, Filzmaier K, Mischke S, et al. Hartmann a potential applications of home monitoring in pacemaker therapy-a review with emphasis on atrial fibrillation and congestive heart failure. Prog Biomed Res 2001;2: $107-114$.

13. Saxon LA, Hayes DL, Gilliam FR, et al. Long-term outcome after ICD and CRT implantation and influence of remote device follow-up: The ALTITUDE survival study. Circulation 2010;122:2359-2367. Epub 2010 Nov 22.

14. Hauck M, Bauer A, Voss F, et al. Home monitoring for early detection of implantable cardioverter-defibrillator failure. Clin Res Cardiol 2009;98:19-24.

15. Res JC, Theuns DA, Jordaens L. The role of remote monitoring in the reduction of inappropriate implantable cardioverter defibrillator therapies. Clin Res Cardiol 2006;95(Suppl 3):III17-21.

16. Lazarus A. Remote, wireless, ambulatory monitoring of implantable pacemakers, cardioverter defibrillators, and cardiac resynchronization therapy systems: Analysis of a worldwide database. Pacing Clin Electrophysiol 2007;30(Suppl 1):S2-12.

17. Joseph GK, Wilkoff BL, Dresing T, et al. Remote interrogation and monitoring of implantable cardioverter defibrillators. J Interv Card Electrophysiol 2004;11:161-166.

18. Ricci RP, Morichelli L, Santini M. Home monitoring remote control of pacemaker and ICD patients in clinical practice. Impact on medical management and health care resource utilization. Europace 2008;10:164-170.

19. Przybylski A, Zakrzewska-Koperska J, Maciag A, et al. Technical and practical aspects of remote monitoring of implantable cardioverter-defibrillator patients in PolandPreliminary results. Kardiol Pol 2009;67:505-511.

20. Fauchier L, Sadoul N, Kouakam C, et al. Potential cost savings by telemedicine-assisted longterm care of implantable cardioverter defibrillator recipients. Pacing Clin Electrophysiol 2005;28:S255-259.

21. Camm J, Kirchhof P, Lip G, et al. Guidelines for the management of atrial fibrillation. The Task Force for the Management of Atrial Fibrillation of the European Society of Cardiology (ESC). Eur Heart J 2010;31:2369-2429.

22. Moya A, Sutton $R$, Ammirati F, et al. Guidelines for the diagnosis and management of syncope (version 2009): The Task Force for the diagnosis and management of syncope of the European Society of Cardiology (ESC). Eur Heart J 2009:30:2631-2671.

23. Calkins H, Brugada J, Packer DL, et al. HRS/EHRA/ECAS expert consensus statement on catheter and surgical ablation of atrial fibrillation. Europace 2007;9:335-379.

24. Jabaudon D, Sztajzel J, Sievert K, et al. Usefulness of ambulatory 7-day ECG monitoring for the detection of atrial fibrillation and flutter after acute stroke and transient ischemic attack. Stroke 2004;35:1647-1651.

25. Hindricks G, Piorkowski $\mathrm{C}$, Tanner $\mathrm{H}$, et al. Perception of atrial fibrillation before and after radiofrequency catheter ablation: Relevance of asymptomatic arrhythmia recurrence. Circulation 2005;112:307-313.

26. Ziegler PD, Koehler JL, Mehra R. Comparison of continuous versus intermittent monitoring of atrial arrhythmias. Heart Rhythm 2006;3:1445-1452.

27. Naccarelli G. Ambulatory electrocardiographic monitoring. J Cardiovasc Electrophysiol 2007;18:248-249.

28. Brignole M, Menozzi C, Bartoletti A, et al. A new management of syncope: Prospective systematic guideline-based evaluation of patients referred urgently to general hospitals. Eur Heart J 2006;27:76-82. 
29. Hindricks G, Pokushalov E, Urban L, on behalf of the XPECT study investigators. Performance of a new leadless implantable cardiac monitor in detecting and quantifying atrial fibrillation. Circ Arrhythm Electrophysiol 2010:3:141-147.

30. Kirchhof P, Auricchio A, Bax J, et al. Outcome parameters for trials in atrial fibrillation: Executive summary. Eur Heart J 2007;28:2803-2817.

31. Joshi AK, Kowey PR, Prystowsky EN, et al. First experience with a mobile cardiac outpatients telemetry (MCOT) system for diagnosis and management of cardiac arrhythmia. Am J Cardiol 2005;95:878-881.

32. Brignole $M$, Vardas $P$, Hoffman $E$, et al. Indications for the use of diagnostic implantable and external ECG loop recorders. Europace 2009;11:671-687.

33. Botto GL, Santini M, Padeletti L, et al. Presence and duration of atrial fibrillation detected by continuous monitoring crucial implications for the risk of thromboembolic events. J Cardiovasc Electrophysiol 2009;20:241-248.

34. Paruchuri V, Adhaduk M, Garikipati NV, et al. Clinical utility of a novel wireless implantable loop recorder in the evaluation of patients with unexplained syncope. Heart Rhythm 2011;8:858-863.

35. Edvardsson N, Frykman V, van Mechelen R, on behalf of the PICTURE study investigators. Use of an implantable loop recorder to increase the diagnostic yield in unexplained syncope: Results from the PICTURE registry. Europace 2010;19:1-8.

36. Huikuri HV, Raatikainen MJP, Moerch-Joergensen R, et al. Prediction of fatal or near fatal cardiac arrhythmia events in patients with depressed left ventricular function after acute myocardial infarction. Results of the cardiac arrhythmias and risk stratification after acute myocardial infarction (CARISMA) study. Eur Heart J 2009;10:109.

37. Spencker S, Coban N, Koch L, et al. A potential role of home monitoring to reduce inappropriate shocks in implantable cardioverter-defibrillator patients due to lead failure. Europace 2009:11:483-488.

38. Heidbuchel $\mathrm{H}$, Lioen $\mathrm{P}$, Foulon $\mathrm{S}$, et al. Potential role of remote monitoring for scheduled and unscheduled evaluations of patients with an implantable defibrillator. Europace 2008:10:351-357

39. Elsner C, Sommer P, Piorkowski C, et al. A prospective multicenter comparison trial of home monitoring against regular follow-up in MADIT II. Comput Cardiol 2006;33:241-244.

40. Schmidt S, Schuchert A, Krieg T. Home telemonitoring in patients with chronic heart failure: A chance to improve patient care? Dtsch Arztebl Int 2010;107:131-138. Epub 2010 February 26.

41. Crossley G, Boyle A, Vitense $\mathrm{H}$, et al. The clinical evaluation of remote notification to reduce time to clinical decision (CONNECT) trial. Am Heart J 2008;156:840-846.

42. Neuzil P, Taborsky M, Holy F, et al. Early automatic remote detection of combined lead insulation defect and ICD damage. Europace 2008;10:556-557.

43. Spencker S, Mueller D, Marek A, et al. Severe pacemaker lead perforation detected by an automatic homemonitoring system. Eur Heart J 2007;28:1432.

44. Ritter O, Bauer WR. Use of "IEGM online" in ICD patients-erarly detection of inappropriate classified ventricular tachycardia via home monitoring. Clin Res Cardiol 2006;95:368-372.

45. Varma N, Stambler B, Chun S. Detection of atrial fibrillation by implanted devices with wireless data transmission capability. Pacing Clin Electrophysiol 2005;28(Suppl 1):S133-136.

46. Varma N. Remote monitoring for advisories: Automatic early detection of silent lead failure. Pacing Clin Electrophysiol 2009;32:525-527.
47. Varma N, Michalski J, Epstein AE, et al. Automatic remote monitoring of ICD lead and generator performance: The TRUST trial. Circ Arrhythm Electrophysiol 2010;3:428-436.

48. Varma N, Epstein A, Irimpen A, et al. The lumos-T safely reduces routine efficacy and safety of automatic remote monitoring for implantable cardioverter defibrillator follow-up. The lumos-T safely reduces routine office device follow-up (TRUST) trial. Circulation 2010;122:325-332.

49. Hauser RG, Hayes DL, Epstein AE, et al. Multicenter experience with failed and recalled implantable cardioverterdefibrillator pulse generators. Heart Rhythm 2006;3: 640-644.

50. Maisel WH. Pacemaker and ICD generator reliability: Metaanalysis of device registries. JAMA 2006;295:1929-1934.

51. Hauser RG, Hayes DL, Almquist AK, et al. Unexpected ICD pulse generator failure due to electronic circuit damage caused by electrical overstress. Pacing Clin Electrophysiol 2001;24:1046-1054.

52. Varma N, Johnson MA. Prevalence of cancelled shock therapy and relationship to shock delivery in recipients of implantable cardioverter-defibrillators assessed by remote monitoring. Pacing Clin Electrophysiol 2009;32(Suppl 1):S42-46.

53. Varma N, Wilkoff BL. Device features for heart failure. Heart Fail Clin North Am 2011;7:215-225.

54. Mortara A, Pinna GD, Johnson P, et al. Home telemonitoring in heart failure patients: The $\mathrm{HHH}$ study (home or hospital in heart failure). Eur Heart J 2009;11:312-318.

55. Saxon LA, Boehmer JP, Neuman S, et al. Remote active monitoring in patients with heart failure (RAPID-RF): Design and rationale. J Cardiac Fail 2007;13:241-246.

56. Yu CM, Wang $\mathrm{L}$, Chau $\mathrm{E}$, et al. Intrathoracic impedance monitoring in patients with heart failure: Correlation with fluid status and feasibility of early warning preceding hospitalization. Circulation 2005;112:841-848.

57. Ritzema J, Melton IC, Richards AM, et al. Direct left atrial pressure monitoring in ambulatory heart failure patients: Initial experience with a new permanent implantable device. Circulation 2007;116:2952-2959.

58. Abraham WT, Adamson PB, Bourge RC, on behalf of the CHAMPION trial study group. Wireless pulmonary artery haemodynamic monitoring in chronic heart failure: A randomised controlled trial. Lancet 2011:377:658-666.

59. Cheung JW, Keating RJ, Stein KM, et al. Newly detected atrial fibrillation following dual chamber pacemaker implantation. J Cardiovasc Electrophysiol 2006;17:13231328 .

60. Glotzer TV, Hellkamp AS, Zimmerman J, et al. Atrial high rate episodes detected by pacemaker diagnostics predict death and stroke. Circulation 2003;107:1614-1619.

61. Glotzer TV, Daoud EG, Wyse DG, et al. The relationship between daily atrial tachyarrhythmia burden from implantable device diagnostics and stroke risk. The TRENDS study. Circ Arrhythmia Electrophysiol 2009;2:474-480.

62. The IMPACT of Biotronik home monitoring guided anticoagulation on stroke risk in patients with implanted ICD and CRT-D devices. NCT00559988@clinicaltrials.gov.

63. Hanke T, Charitos EI, Stierle U, et al. Twenty-four-hour holter monitor follow-up does not provide accurate heart rhythm status after surgical atrial fibrillation ablation therapy. Circulation 2009;120(Suppl 11):S177-184.

64. Halimi F, Cle'menty J, Attuel $\mathrm{P}$, et al., on behalf of the OEDIPE trial investigators. Optimized post-operative surveillance of permanent pacemakers by home monitoring: The OEDIPE trial. Europace 2008;10:1392-1399. Epub 2008 September 4

65. Mabo P, Victor F, Bazin P, et al. Home monitoring for pacemaker follow-up: Results of randomized COMPAS trial. Europace 2010;12(S1):i56. 
66. Ricci RP, Morichelli L, Quart A, et al. Long-term patient acceptance of and satisfaction with implanted device remote monitoring. Europace 2010;12:674-679.

67. Kallinen LM, Hauser RG, Tang C, et al. Lead integrity alert algorithm decreases inappropriate shocks in patients who have Sprint Fidelis pace-sense conductor fractures. Heart Rhythm 2010;7:1048-1055.

68. Simons EC, Feigenblum DY, Nemirovsky D, et al. Alert tones are frequently inaudible among patients with implantable cardioverter-defibrillators. Pacing Clin Electrophysiol 2009;32:1272-1275.

69. Hauser RG, Hayes DL, Epstein AE, et al. Multicenter experience with failed and recalled implantable cardioverterdefibrillator pulse generators. Heart Rhythm 2006;3 640-644.

70. Brugada P. What evidence do we have to replace in-hospital implantable cardioverter defibrillator follow-up? Clin Res Cardiol 2006;95:iii/3-9.

71. Raatikainen MJ, Uusimaa P, van Ginneken MM, et al. Remote monitoring of implantable cardioverter defibrillator patients: A safe, time-saving, and cost-effective means for follow-up. Europace 2008;10:1145-1151.

72. Halperin D, Heydt-Benjamin TS, Ransford B, et al. Pacemakers and implantable cardiac defibrillators: Software radio attacks and zero-power defenses. Proceedings of the 2008 IEEE Symposium on Security and Privacy 2008; $129-142$.

73. Wireless ICD programming vulnerable to hackers, report claims. http://www.theheart.org/article/847781.do (accessed date 13 March 2011).

74. Gue'don-Moreau L, Chevalier P, Marquié C, et al. Contributions of remote monitoring to the follow-up of implantable cardioverter-defibrillator leads under advisory. Eur Heart J 2010;31:2246-2252.

75. Sticherling $C$, Kühne $M$, Schaer $B$, et al. Remote monitoring of cardiovascular implantable electronic devices. Prerequisite or luxury? Swiss Med Wkly 2009;139: 596-601.

76. Neumann PJ, Tunis SR. Medicare and medical technologythe growing demand for relevant outcomes. N Engl J Med 2010;362:377-379.

77. Colechin E, Reay C, Bousfiled D, et al. Implantable cardiac devices with remote monitoring facilities. NHS Purchasing and Supply Agency, Centre for Evidencebased Purchasing. 2009. Policy and Innovation Directorate of the NHS Purchasing and Supply Agency, CEP09010.

78. Vinck I, Stroobandt S, Gerkens S, et al. Remote Monitoring for Patients with Implanted Defibrillators. Brussels/Belgium, The Belgian Health Care Knowledge Centre, 2010.

79. Medical Services Advisory Committee. Remote monitoring systems for patients with implanted cardiac devices; June 2008. MSAC Application 1111. Retrieved on February 2011 from: http://www.health.gov.au/internet/msac/publishing. nsf/Content/115CC907F00447B3CA2575AD0082FD6C/ \$File/MSAC\%201111\%20Report_FINAL\%2028\% 20October\%202008.pdf

80. Klersy C, De Silvestri A, Gabutti G, et al. A meta-analysis of remote monitoring of heart failure patients. J Am Coll Cardiol 2009;54:1683-1694.

81. Trueman P, Grainger DL, Downs KE. Coverage with evidence development: Applications and issues. Int J Technol Assess Health Care 2010;26:179-185. 